\title{
La publicidad interactiva en España: situación actual y tendencias
}

\section{Antonio Baladrón Pazos y Clara Muela Molina}

\section{Resumen}

Este artículo profundiza en la situación actual y las tendencias de la industria publicitaria en Internet en España. Se exponen los datos más actuales sobre los diferentes agentes implicados: el propio medio, los anunciantes, las agencias, las audiencias y los productos creativos.

\section{Palabras clave}

Anunciantes. Agencias interactivas.

Publicidad interactiva. Internet. España.
Antonio Baladrón Pazos I antonio.baladron@urjc.es Doutor en Publicidade e RR.PP. pela Universidade de Vigo. Atualmente pesquisador e professor la Universidade Rey Juan Carlos de Madrid.

Clara Muela Molina I clara.muela@urjc.es

Doutora em CC. da Informação pela Universidad Complutense Professora da Universidad Rey Juan Carlos (Madrid, Espanha).

\section{Introducción}

Internet ha abierto nuevos horizontes para

la publicidad, a menudo aquejada de falta de eficacia y de excesiva unilateralidad. El avance de la creatividad en la Red (MUELA MOLINA, 2008b) ha sido tan destacado que en la actualidad los profesionales españoles ocupan puestos preferentes en los ranking de las principales empresas o campañas del sector de la publicidad digital. En las próximas líneas no proponemos un análisis centrado exclusivamente en los mensajes o campañas publicitarias - pues dicha tarea correspondería con más legitimidad a los propios creativos y profesionales que han participado en su planificación y desarrollo -, sino una radiografía del contexto global de la industria publicitaria en Internet. Sólo así podremos comprender el alcance que está cobrando este ámbito y su constante adaptación al cambio, obligado por el incesante desarrollo de la tecnología.

Para abordar este objeto de estudio, lo primero que haremos será aproximarnos al medio desde el punto de vista de las cifras de inversión 
publicitaria que las empresas destinan a él.

En segundo lugar, expondremos cuáles son los principales anunciantes, refiriendo los sectores que más confían sus presupuestos publicitarios a este medio. En tercer lugar, mencionaremos la relevancia que han adquirido las agencias especializadas en publicidad interactiva españolas en el mundo. En cuarto lugar, recogeremos algunos datos y reflexiones acerca de los principales formatos, campañas y tendencias en la publicidad interactiva. Y, finalmente, analizaremos las audiencias de Internet y la percepción que tienen de los mensajes comerciales que se difunden a través de la Red. Se trata, en suma, de realizar un recorrido por los diferentes agentes implicados en esta industria publicitaria, desde el propio medio y hasta los anunciantes, las agencias o las audiencias, sin olvidar, por supuesto, la relación de todos ellos con los formatos publicitarios más empleados.

\section{Un medio publicitario en crecimiento}

No cabe duda de que Internet es un medio que se ha ido consolidando como una apuesta atractiva para pequeños y grandes anunciantes. Según la consultora Infoadex (2009, p. 21), fue el quinto medio por volumen de inversión en 2008, tal y como se aprecia en el Gráfico 1; con un porcentaje de un $8,6 \%$ sobre el total, sube una posición con respecto al año anterior, en el que ese quinto puesto era ocupado por el medio exterior.

Durante el pasado año, los anunciantes gastaron en España 14.915,3 millones de euros en publicidad, un 7,5\% menos sobre los 16.121,3 millones de 2007. El 47,6\% se destinó a medios convencionales, en concreto 7.102,5 millones; en el contexto de esa partida Internet se muestra como un medio muy importante, superando en inversión a otros como cine, dominicales 0 exterior, y situándose a tan sólo

Gráfico 1- Inversión publicitaria real estimada: distribución en medios convencionales 2008 en \%
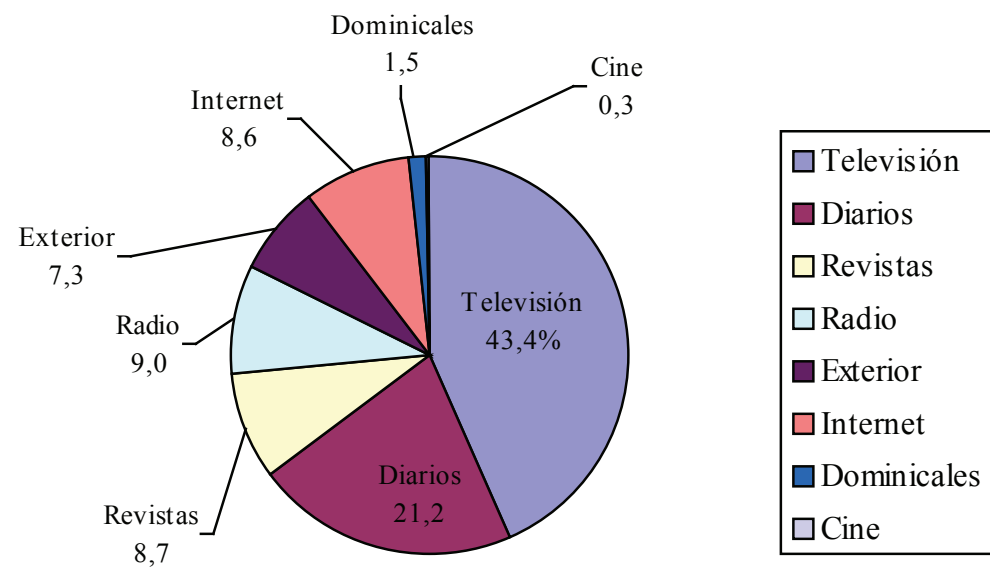

Fuente: Infoadex/ IAB 
una décima del medio revistas 0 a apenas cuatro de la radio. Además, los últimos datos constatan que tiene el mayor índice de crecimiento porcentual con respecto al resto de medios convencionales: televisión, diarios, revistas, radio, exterior, dominicales y cine; es, de hecho, el único que ha obtenido un crecimiento positivo en 2008 .

Eso explica que, según Infoadex (2009, p. 24) y de acuerdo con las cifras recogidas en la Tabla 1, los principales anunciantes otorguen una gran confianza a Internet - a pesar de ser relativamente joven y estar en constante evolución - y apuesten por él de forma cada vez más firme y decidida.

Match.com o Meetic son, según los datos de participación de Internet en el conjunto de inversiones publicitarias (INFOADEX, 2009, p. 291), algunas muestras de esa apuesta por la Red que hacen las empresas de base tecnológica; el grueso de su inversión va destinado a Internet, por lo que actualmente ocupan el séptimo y octavo lugar por inversión, respectivamente.

Asimismo, anunciantes tradicionales, que se han ido adaptando a un contexto mediático en constante cambio, valoran cada vez más este medio; no en vano el $79 \%$ de las empresas que planifican sus campañas en Internet son anunciantes tradicionales, como recoge el estudio Análisis de la publicidad on-line en España, realizado en abril de 2009 por Trafficking

\section{Solutions Hispania.}

Partiendo de este mismo estudio, se puede afirmar que la evolución del comportamiento del anunciante digital se traduce en una disminución de las campañas de autopromoción, una estabilización en las de lanzamiento de nuevos productos y un considerable incremento en las de marca. El 15,8\% de las campañas contiene una promoción y el 37,3\% son de lanzamiento de productos o servicios; hay que tener en cuenta que en 2006 éstas últimas suponían sólo el 14\%, mientras que en 2007 ya representaban el $35 \%$, de modo que se puede deducir una clara

Tabla 1- Inversión publicitaria real estimada: facturación anual medios convencionales 2002-2008 en millones de euros.

\begin{tabular}{l|c|c|c|c|c|c|c|c|}
\hline & $\mathbf{2 0 0 2}$ & $\mathbf{2 0 0 3}$ & $\mathbf{2 0 0 4}$ & $\mathbf{2 0 0 5}$ & $\mathbf{2 0 0 6}$ & $\mathbf{2 0 0 7}$ & Var. & $\mathbf{2 0 0 8}$ \\
\hline Televisión & $2.172,2$ & $2.317,2$ & $2.669,9$ & $2.951,4$ & $3.188,4$ & $3.467,0$ & $-11,1$ & $3.082,1$ \\
\hline Diarios & $1.531,2$ & $1.496,0$ & $1.583,7$ & $1.666,4$ & $1.790,5$ & $1.894,4$ & $-20,4$ & $1.507,9$ \\
\hline Radio & 484,9 & 508,2 & 540,2 & 609,9 & 636,7 & 678,1 & $-5,3$ & 641,9 \\
\hline Revistas & 590,1 & 601,2 & 664,3 & 674,6 & 688,1 & 721,8 & $-14,5$ & 617,3 \\
\hline Internet & $\mathbf{7 1 , 2}$ & $\mathbf{7 2 , 6}$ & $\mathbf{9 4 , 6}$ & $\mathbf{1 6 2 , 4}$ & $\mathbf{3 1 0 , 5}$ & $\mathbf{4 8 2 , 4}$ & $\mathbf{2 6 , 5}$ & $\mathbf{6 1 0 , 0}$ \\
\hline Exterior & 408,6 & 454,0 & 474,3 & 493,9 & 529,1 & 568,0 & $-8,8$ & 518,3 \\
\hline Dominicales & 106,8 & 105,9 & 110,0 & 119,3 & 123,2 & 133,5 & $-22,2$ & 103,9 \\
\hline Cine & 45,3 & 47,6 & 40,7 & 42,9 & 40,6 & 38,4 & $-45,4$ & 21,0 \\
\hline Total & $5.410,3$ & $5.602,7$ & $6.177,7$ & $6.720,8$ & $7.307,1$ & $7.983,6$ & $-11,1$ & $7.102,5$ \\
\hline
\end{tabular}


consolidación y continuismo de la inversión en este tipo de publicidad. Por su parte, el 46\% de las campañas en Internet son de branding 0 comunicación de marca.

\section{Los anunciantes apuestan por Internet}

Deteniéndonos un poco más en los anunciantes que orientan sus presupuestos publicitarios hacia Internet, cabe destacar que empresas tradicionales de sectores como la banca, la automoción o el turismo están comprobando la extraordinaria eficacia de sus comunicaciones on-line. Tras la crisis sufrida a finales de los años 90 por las empresas .com, Internet ha ido ganando adeptos entre anunciantes que apenas conocían ni utilizaban el medio, al tiempo que ha recuperado la confianza perdida por el resto de anunciantes. Actualmente se puede decir que las empresas de los sectores mencionados, entre otras, han puesto en valor el medio, descubriendo las posibilidades creativas que tiene y constatando su potencial comunicativo con el consumidor.
Prueba de ello es la apuesta que en nuestro país hacen por la Red anunciantes con grandes presupuestos publicitarios (INFOADEX, 2009, p. 44). Es el caso de la multinacional de las telecomunicaciones Telefónica, que lidera el ranking general de inversiones en publicidad en España con un gasto de 173 millones de euros durante 2008 y que ocupa el tercer puesto en el listado de inversores en Internet. En la Tabla 2 se pueden observar comparativamente las posiciones que ostentan los principales anunciantes en el ranking de total de medios y en el de Internet. Por poner algún otro ejemplo, Vodafone o ING Direct, que se sitúan respectivamente en los puestos quinto y noveno, están en el $11^{\circ}$ y primero en el medio Internet. Mientras tanto, otras grandes empresas siguen prefiriendo medios clásicos como la televisión 0 la radio porque consideran que les reportan más notoriedad; así, Procter \& Gamble España, L'Oreal España o los grandes almacenes $E l$ Corte Inglés apenas utilizan Internet y sin embargo en el ranking de inversión total en

Tabla 2- Comparativa ranking anunciantes por inversión 2008: posición total medios/total Internet

\begin{tabular}{|c|l|c|}
\hline Total Medios & Anunciante & Total Internet \\
\hline 1 & Telefónica, S.A.U. & 3 \\
\hline 5 & Vodafone España, S.A. & 11 \\
\hline 6 & Volkswagen-Audi España, S.A. & 18 \\
\hline 7 & Renault España Comercial, S.A. & 5 \\
\hline 9 & ING Direct, N.V., España & 1 \\
\hline 10 & France Telecom España, S.A. & 9 \\
\hline 16 & Ford España, S.L. & 13 \\
\hline 17 & Automóviles Citröen España, S.A. & 14 \\
\hline
\end{tabular}


medios ocupan, por este orden, las posiciones segunda, tercera y cuarta.

Según Infoadex (2009, p. 291), de los diez primeros puestos del ranking de anunciantes en Internet, siete lo son de los sectores de Finanzas, Telecomunicaciones e Internet. Así mismo, marcas como Endesa, Ebay, Interflora o Hachette desaparecen del listado de los 20 anunciantes con mayor inversión; sólo esos 20 facturan el 31,9\% de los ingresos publicitarios en la Red. El liderazgo lo tiene, como ya hemos adelantado, ING Direct, con un gasto de 17,3 millones de euros frente a los 10,4 de 2007. A una distancia considerable se sitúan el periódico digital Segunda Mano (7,8 millones), Telefónica $(7,8)$ y Banco Español de Crédito $(7,4)$; el siguiente es Renault, con un gasto de 6,2 millones, y tras él otros con inversiones que rondan $\operatorname{los} 4$ millones.
Comparando estos datos con los del año anterior, la conclusión más significativa es la continuación del liderazgo de $I N G$ Direct y la presencia entre los veinte primeros puestos de muchas de las marcas que ya estaban en el ranking de 2007, si bien variando el orden. Por otro lado, entre los anunciantes que descienden posiciones cabe destacar Match.com, que baja 7, Meetic 4, Ford 8 y Vodafone 4. Por el contrario, otras empresas suben posiciones, como Telefónica, que asciende 5 puestos.

Para un más exhaustivo acercamiento a los principales sectores de actividad de los anunciantes en Internet, es necesario hacer referencia al Estudio de inversión en medios interactivos en España 2008, realizado por PricewaterhouseCoopers para el Interactive Advertising Bureau (www.iabspain.net). Como

Gráfico 2- Distribución de ingresos por la actividad del anunciante de 2005 a 2008 en \%

$$
\square 2005 \quad \square 2006 \quad \square 2007 \quad \square 2008
$$

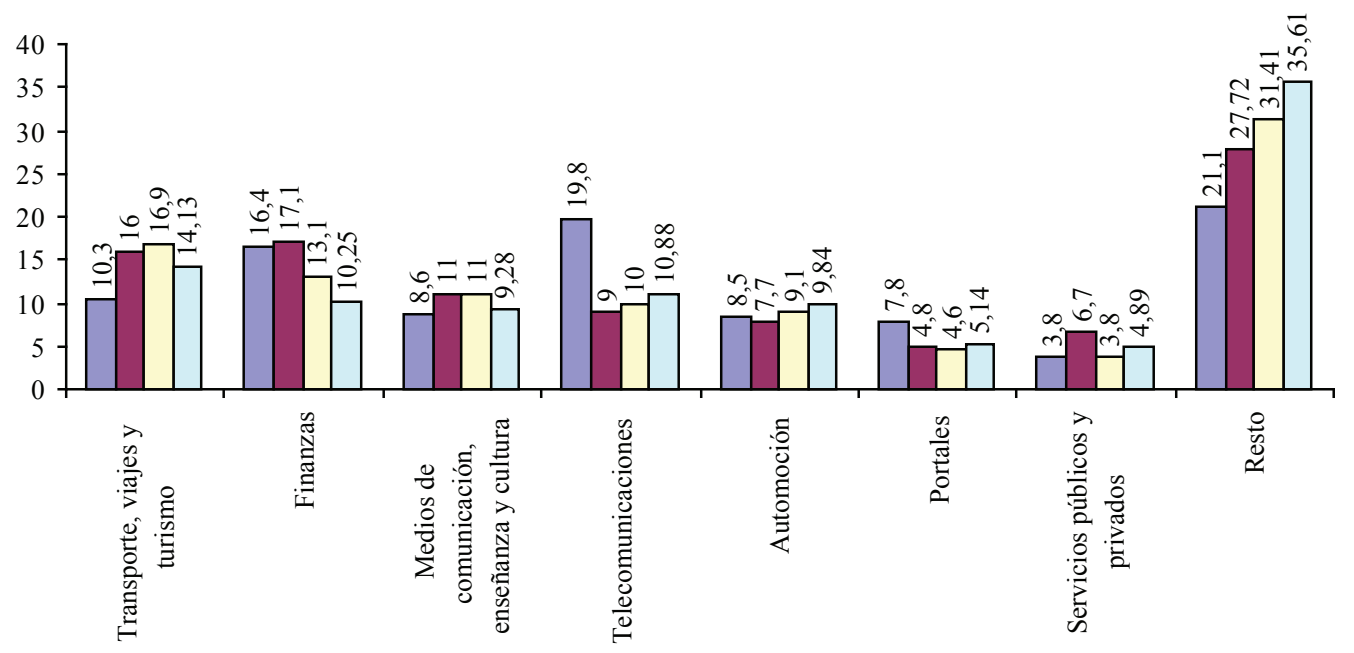


se recoge en el Gráfico 2, el 54,38\% de la inversión es acaparada por cinco sectores: Transporte, viajes y turismo (que representa el 14,13\%); Telecomunicaciones (el 10,88\%); Finanzas (10,25\%); Automoción (9,84\%); y Medios de comunicación, enseñanza y cultura (9,28\%). Muy por debajo están los sectores de Portales $(5,14 \%)$ y Servicios públicos y privados (4,89\%). Los restantes quince sectores apenas suponen en su conjunto un tercio de la inversión.

Es importante subrayar que la mayor parte de los sectores controlados en dicho estudio aumenta su inversión con respecto a 2007 (www. iabspain.net), lo cual corrobora la confianza que los anunciantes ya tienen en el medio. Recorta un -2,85\% Finanzas, sector que ya había experimentado una caída presupuestaria en el año 2006. Por su parte, también cae el presupuesto de dos sectores vinculados al ámbito del ocio, como son Transportes, viajes y turismo y Medios de comunicación, enseñanza y cultura; en ambos casos el decremento rompe la inercia de los tres últimos años, en los que tendían a estabilizarse.

Finalmente (véase Tabla 3), existe una clara tendencia a la estabilización de determinados anunciantes en los puestos de privilegio de los listados de empresas que más invierten en publicidad on-line. Tanto en el ranking de inversores en otros medios como en el general que realiza Infoadex se da una un inercia por parte de las marcas a ocupar prácticamente los mismos escalones, con ligeras diferencias de un año para otro.

\section{Liderazgo español entre las agencias interactivas}

Junto al estudio del anunciante, para comprender la industria publicitaria en Internet en España es necesario hacer referencia a la realidad actual y el alcance de las agencias interactivas. No existe un ranking de las principales empresas publicitarias por volumen de inversión, pero sí puede ser útil la lista de las agencias interactivas más premiadas a nivel mundial según The Won Report 2008 (www.thebigwon.com). Este ranking pone en evidencia el importante papel de las agencias con sede en España, ya que el liderazgo es ocupado por la madrileña Shackleton. Además, en séptimo lugar se sitúa otra española, $C P$ Proximity, que sube puestos con respecto a la $12^{\mathrm{a}}$ posición del año anterior. La tercera agencia con sede en España, $16^{\text {a }}$ a nivel mundial, es OgilvyOne Spain.

\section{La interactividad sigue siendo un reto}

Tras abordar el anunciante y la agencia como actores esenciales del negocio publicitario, conviene ahora profundizar en algunas de las más importantes características y retos de la publicidad digital. La interactividad sigue siendo una de sus principales ventajas y los anunciantes están sabiendo utilizarla para la 
Tabla 3- Ranking de los 50 primeros anunciantes en publicidad gráfica 2008-7

\begin{tabular}{|c|c|c|c|c|c|c|}
\hline & Anunciante 2008 & Anunciante 2007 & Sector & & Anunciante 2008 & Anunciante 2007 \\
\hline 1 & Iberia & Vueling & \multirow{5}{*}{$\begin{array}{c}\text { ense } \\
\tilde{\text { nanz }} \\
\mathbf{a} \mathbf{y} \\
\text { cultu } \\
\text { ra }\end{array}$} & 6 & Warnes Bross & Disney \\
\hline 2 & Vueling & Iberia & & 7 & Universal Pict & El País \\
\hline 3 & E-Dreams & Spanair & & 8 & El País & Prisa \\
\hline 4 & Clickair & Clickair & & 9 & MTV & Filmax \\
\hline 5 & Atrápalo & Mucho viaje & & 10 & Canal S. Digi & La Sexta \\
\hline 6 & Viajes El C. Inglés & E-Dreams & \multirow{10}{*}{$\begin{array}{c}4 . \\
\text { Telec } \\
\text { omu } \\
\text { nicac } \\
\text { iones }\end{array}$} & 1 & Vodafone & Vodafone \\
\hline 7 & Muchoviaje & Bookings.com & & 2 & Telefónica Móv. & Telefónica \\
\hline 8 & Bookings & Terminal A & & 3 & France Telecom & Orange \\
\hline 9 & Lufthansa & RENFE & & 4 & Telefónica & Telefónica Móv. \\
\hline $\begin{array}{l}1 \\
0\end{array}$ & Spanair & Viajes El C. Inglés & & 5 & Simyo & $\mathrm{ONO}$ \\
\hline 1 & ING Direct & ING Direct & & 6 & KPN & Telecom. \\
\hline 2 & Banesto & Banco Cetelem & & 7 & Yoigo & Yoigo \\
\hline 3 & BBVA & La Caixa & & 8 & ONO & Nokia \\
\hline 4 & Caja Madrid & Banesto & & 9 & Movilisto & Jazztel \\
\hline 5 & $\mathrm{BSCH}$ & Banco Sygma & & 10 & Nokia & Tele 2 \\
\hline 6 & Bancaja & Cortal Consors & \multirow{10}{*}{$\begin{array}{c}5 . \\
\text { Auto } \\
\text { moci } \\
\text { ón }\end{array}$} & 1 & Ford & Peugeot \\
\hline 7 & Société G. Bank & BBVA & & 2 & Renault & Renault \\
\hline 8 & La Caixa & Uno-e Bank & & 3 & Peugeot & Ford \\
\hline 9 & Uno-eBank & $\mathrm{BSCH}$ & & 4 & General Motors & Volkswagen Audi \\
\hline $\begin{array}{l}1 \\
0\end{array}$ & CAM & Bancaja & & 5 & Volkswagen & Toyota \\
\hline 1 & Fox & CEAC & & 6 & Nissan & General Motors \\
\hline 2 & CEAC & Fox & & 7 & Mercedes Benz & Kia \\
\hline 3 & Planeta Directo & Warner BROS & & 8 & Daimler Chrysler & Citroen \\
\hline 4 & Antena 3 & Planeta Directo & & 9 & Toyota & Opel \\
\hline 5 & Buena Vista Int. & Universal Pictures & & 10 & BMW & Seat \\
\hline
\end{tabular}

Fuente: IAB-PwC

\begin{tabular}{|l|l|c|}
\hline & Agencia & Puntos \\
\hline $\mathbf{1}$ & Shackleton, Madrid & 279 \\
\hline $\mathbf{2}$ & CP Comunicacion Argentina & 84 \\
\hline $\mathbf{3}$ & Mark/M\&C Saatchi, Sydney & 78 \\
\hline $\mathbf{4}$ & Heye \& Partner, Hamburg & 72 \\
\hline $\mathbf{4}$ & Proximity London & 72 \\
\hline $\mathbf{6}$ & Salem Sao Paulo & 70 \\
\hline $\mathbf{7}$ & CP Proximity Spain & 67 \\
\hline $\mathbf{8}$ & Jung von Matt, Hamburg & 66 \\
\hline $\mathbf{9}$ & Ogilvy Frankfurt & 64 \\
\hline $\mathbf{9}$ & BMF Sydney & 64 \\
\hline $\mathbf{1 1}$ & Lowe + Rivet, Australia & 60 \\
\hline
\end{tabular}


consecución de sus objetivos, si bien todavía debieran desarrollarla más; de hecho, el feedback simultáneo entre emisor y receptor podría ser mejor aprovechado en aspectos como la solicitud de información, la gestión de compra y distribución o incluso la atención más inmediata a las sugerencias y quejas de los consumidores.

Por mímesis con otros medios, muchas campañas siguen integrándose en las páginas web de forma bastante artificial en lugar de adaptarse al lenguaje específico hipertextual del medio. Prueba de ello son formatos como los banner, botones, robapáginas o rascacielos, ubicados en las zonas más destacadas de las páginas y que se presentan como una traslación a Internet de las prácticas y procedimientos habituales en medios tradicionales. El resultado es una publicidad que a grandes rasgos continúa siendo demasiado intrusiva, molesta, que constantemente interrumpe la navegación del usuario.

Frente a ella, se impone un tipo de publicidad más respetuosa y selectiva cuyo fin es aportar valor al consumidor en lugar de imponerse sin permiso, una publicidad útil, ajustada a la demanda de los navegantes, eficaz por tanto. No en vano cada vez se contratan menos los formatos intrusivos y más los que implican un complemento a la navegación, como es el caso de los relacionados con el marketing de buscadores. Así mismo, los principales motores del crecimiento de la publicidad interactiva en los últimos años son, por una parte, la creciente apuesta de los anunciantes en la mayoría de los sectores, pero también, por otra, el modelo de buscadores y enlaces patrocinados ${ }^{1}$, que son los formatos preferidos por muchas empresas.

Ese ascenso de la inversión se observa, además de en los datos referidos en epígrafes anteriores, en las cifras del IAB sobre inversión en publicidad on-line en 2008 (www.iabspain.net), que alcanzan ya los 610,04 millones de euros, 127,62 millones más que en 2007, en términos porcentuales un incremento respecto al año pasado del $26,45 \%$. Hay que tener en cuenta que el IAB e Infoadex llegaron, en 2007, a un acuerdo según el cual a las cifras que venía aportando este último informe acerca de los formatos gráficos se añaden, con carácter retroactivo, las de otras acciones como los enlaces patrocinados.

\subsection{Formatos publicitarios y eficacia ${ }^{2}$}

Precisamente los enlaces patrocinados han experimentado un incremento interanual del

En un artículo publicado el año pasado en la revista Telos se describen, entre otros aspectos, las herramientas utilizadas por los motores de búsqueda, la problemática de Google en este concepto a través de sus "spiders" o la peculiaridad de su sistema de enlaces patrocinados Google Adwords, el sistema más contratado por los anunciantes basado en el Coste Por Click (CPC) para aumentar el tráfico a la web de la empresa contratante; es decir, toda una estrategia publicitaria inspirada en el concepto de posicionamiento y basada en la percepción visual o el recorrido del ojo frente a la pantalla del ordenador (MUELA MOLINA, 2008a).

Todos los datos de inversión referentes a la publicidad interactiva según modalidad de los formatos contratados a lo largo del año 2008 han sido obtenidos del IAB capítulo español, única asociación que ofrece dichos datos anualmente elaborados por la consultora PricewaterhouseCoopers, al igual que en el resto de países donde está presente. 
$36,45 \%$ hasta suponer en 2008 el 53,18\% del

total de la inversión en publicidad interactiva; se destinaron a este formato 322,44 millones de euros. Si comparamos estas cifras con las de los ejercicios anteriores, tal y como se recoge en el Gráfico 3, concluiremos sobre la importantísima apuesta que los anunciantes hacen por este tipo de publicidad; sin duda, debido a su alta rentabilidad y eficacia, que sigue garantizando unos destacados niveles de ROI (Return On Investment) para las empresas que lo utilizan ${ }^{3}$.

Después de los enlaces patrocinados, los formatos integrados son los que reciben mayor inversión (véase Tabla 5). Banner, botones y sellos suben un puesto en el ranking y suponen el 10,66\% del total; a continuación se sitúan los robapáginas y rascacielos, que ceden segundo lugar con un 9,97\%. Le siguen en cuarto y quinto lugar los patrocinios o secciones fijas y las acciones a través del mail. En el primer caso la inversión sube del $3,75 \%$ en 2007 al 4,37\% en 2008, mientras que en el segundo se queda en el 3,44\%, un porcentaje similar al del ejercicio anterior. Muy por debajo, los anunciantes continúan utilizando otras posibilidades como el spot en vídeo (que supone el 1,04\%), los interstitial y supertitial (con una subida poco significativa, pasando del 1,37\% al 1,43\% ) o los layer y otros formatos desplegables (el 1,42\%). Por último, las acciones de marketing a través del móvil siguen por el momento representando un porcentaje residual, un $0,26 \%$, a pesar de las expectativas puestas en ellas por los expertos.

Gráfico 3- Evolución facturación anual de buscadores y enlaces patrocinados 2005/2008 en millones de euros

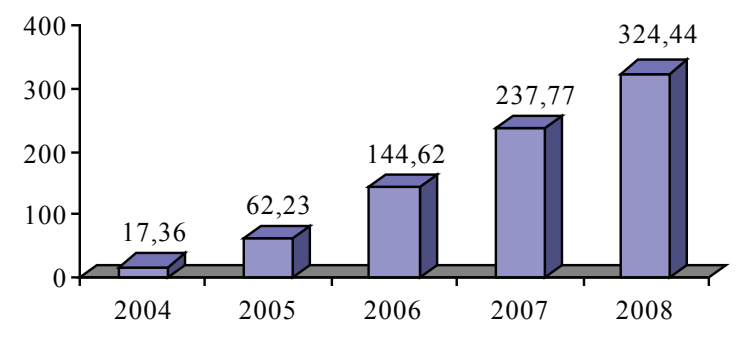

Fuente: IAB Spain

Estas cifras empiezan a parecerse a las resultantes de otros mercados donde la publicidad interactiva está más desarrollada como puede ser Estados Unidos con un 45\% (www.iab.net) o Reino Unido con una cifra más abultada, el 59,3\% (www.iabuk.net). No obstante, cabe hacer dos matizaciones: primero, la cifra resultante de estos dos países sólo refleja la partida presupuestaria del formato buscadores (search) separándola de la de los enlaces patrocinados; y segundo, no hay uniformidad total en las clasificaciones utilizadas para la realización de los estudios de inversión, variando éstas de una zona geográfica a otra, y tampoco los informes se hacen públicos de manera pareja ni en forma ni en tiempo, aún siendo la misma asociación que está presente en la mayoría de países desarrollados. 
Tabla 5- Inversión por tipos de formatos 2007-2008 en \%

\begin{tabular}{l|l|c|c|}
\hline Formato & Tipo & $\mathbf{2 0 0 8 \%}$ & $\mathbf{2 0 0 7 \%}$ \\
\hline \multirow{2}{*}{ Formatos Integrados } & Enlaces patrocinados & 53,18 & 49,29 \\
\cline { 2 - 4 } & Banner, botones y sellos & 10,66 & 9,33 \\
\cline { 2 - 4 } & Robapáginas, rascacielos & 9,97 & 11,17 \\
\hline Patrocinios o secciones fijas & Patrocinios o secciones fijas & 4,37 & 3,75 \\
\hline \multirow{2}{*}{ E-mail } & E-mail & 3,44 & 3,63 \\
\hline Spot en vídeo & Layer y otros formatos desplegables & 1,42 & 1,13 \\
\cline { 2 - 4 } & Por-up, Pop-under & 0,04 & 0,11 \\
\hline Formato de transición & Spot en vídeo & 1,04 & 1,48 \\
\hline Mobile Marketing & Interstitial y Superstitial & 1,43 & 1,37 \\
\hline Advergaming & Mobile Marketing & 0,26 & 0,52 \\
\hline Otros & Advergaming & 0,05 & 0,02 \\
\hline
\end{tabular}

Fuente: IAB Spain

De igual manera, es minoritario el 0,26\% del advergaming, que se estrenó el pasado año con un 0,05\%, y el 0,04\% de las molestas y fácilmente eludibles pop-up у pop-under.

En definitiva, la mayor parte de la inversión va destinada al grupo de los integrados, que son aquellos que se emplazan visualmente en algún lugar de las páginas web; el IAB incluye en este grupo a enlaces patrocinados, banner, botones, sellos, robapáginas y rascacielos. El porcentaje conjunto que representan llega al 73,81\%, bastante próximo al 69,79\% de 2007.

Mientras tanto, los anunciantes confían sus presupuestos publicitarios cada vez menos a los formatos gráficos: los que no están integrados en una página web, como es el caso de las popup y pop-under, los desplegables, formatos de transición, rich media, vía e-mail, etc. En 2006 representaban el 47,53\% de la inversión, en 2007 el 30\% y en 2008 esos porcentajes han descendido aún más, hasta el 26,19\%.

\subsection{Campañas on-line de éxito}

Tal y como se adelantó en el epígrafe 4, la industria de la publicidad interactiva cuenta en España con destacados profesionales a nivel mundial. El prestigio creativo queda de manifiesto en los galardones obtenidos en los últimos años por las agencias ubicadas en nuestro país. Así, en el Festival Internacional de Cannes 2008 España consiguió dos leones de Plata correspondientes a la categoría de Cyber; se trata de la campaña "Eliminate", realizada por Shackleton para Acción contra el Hambre, y "The key to reserva", de la marca catalana Freixenet y realizada por la agencia J. Walter Thompson.

Por su parte, en la sección de acciones publicitarias on-line, categoría de campañas online, del Festival Publicitario Iberoamericano de El Sol,Atrápalo.com se alzó con el Sol de Oro por una campaña publicitaria de Double You: "El primer concurso de televisión que no sucede en televisión". En este mismo certamen, 
la también catalana Villarrosás obtuvo el Sol de Plata con la campaña de Nike para la San Silvestre Vallecana (http://www.doubleyou.com/ sansilvestre/cannes/).

La mencionada campaña de Atrápalo.com (www.doubleyou.com) obtuvo unos resultados muy positivos debido a su carácter innovador de cara a los consumidores; sólo en el primer mes de campaña se consiguieron 90.000 usuarios registrados y el portal de viajes que lo impulsó incrementó su notoriedad de marca en más de un $150 \%$. De hecho, el concurso constituía una clara estrategia para conseguir notoriedad y visibilidad en televisión. Se creó un microespacio televisivo con el nombre de la marca y en el que se explicaban en qué consistía el concurso, cuáles era los premios, participación de concursantes, etc., remitiendo a los interesados a Internet, que era donde verdaderamente se desarrollaba el juego. Además, con ello se perseguía construir la imagen de marca de Atrápalo.com como una agencia de publicidad distinta, interactiva $\mathrm{y}$ atrayente.

Junto al juego, en Internet se puso en marcha una página oficial en la que los navegantes encontraban información sobre la marcha del concurso y todo tipo de utilidades, desde subir sus fotografías para aparecer por televisión en los microespacios que se difundían hasta ver los programas de televisión colgados en Youtube. También se elaboraron banner rich media a través de los cuales los interesados podían entrenarse para el juego.

\section{La audiencia, en constante evolución}

Para terminar este recorrido por la situación actual de la industria publicitaria en Internet nos queda recoger algunas reflexiones sobre las audiencias y la percepción que éstas tienen de Internet como medio publicitario. Según un estudio de la consultora ComScore (www.comscore.com), España ocupa la cuarta posición de los países europeos - empezando el cómputo de la lista por el final - por penetración de Internet con un 39\% de acceso a la Red en los hogares españoles, porcentaje que supera la media de dicho concepto a nivel mundial cifrada en un 18,9\% y nos sitúa en niveles superiores a la media europea. No obstante, en cuanto al índice de personas conectadas a la Red, España se encuentra muy alejada respecto a otros países vecinos como Holanda (82\%), Noruega o Suecia (76\%), Francia (61\%), Inglaterra (65\%) 0 Alemania (49\%). Por eso, pese a los avances tecnológicos y al enorme crecimiento que ha experimentado Internet en los últimos años, los anunciantes siguen viendo en la reducida tasa de penetración uno de los frenos a la hora de decidirse por su utilización. Ahora 
bien, el tiempo dedicado a Internet y la

audiencia ${ }^{4}$ del mismo han ido aumentando año tras año de forma leve pero constante.

\subsection{Hábitos de los usuarios de Internet}

Según la $11^{a}$ edición del estudio Navegantes en la Red (ASOCIACIÓN PARA LA INVESTIGACIÓN

DE MEDIOS DE COMUNICACIÓN, 2009, p. 52;

p. $41 ;$ p. 87), el $26 \%$ de los jóvenes dedica una media de una a dos horas diarias a navegar y el $68,2 \%$ está cada vez menos tiempo frente a la pantalla del televisor y más ante la del ordenador. En cuanto a las actividades que realizan los internautas, tomando como referencia el último mes de uso de Internet, las principales son las búsquedas a través de buscadores y directorios, con un porcentaje del $95,8 \%$, y la lectura de noticias de actualidad, con un $87,8 \%$. En este sentido, en el ranking de las webs más visitadas por los españoles destacan las de medios de comunicación tradicionales: diarios, emisoras de radio o cadenas de televisión; 4 de los 10 primeros puestos corresponden a versiones digitales de diarios tradicionales en papel.

Esto mismo es corroborado por los datos de la Oficina para la Justificación de la Difusión Interactiva (www.ojdinteractiva.com), según los cuales la web del periódico El Mundo es la segunda con mayor número de usuarios y el medio de comunicación con más audiencia en Internet; tiene un total de 20.086.109 usuarios únicos y 340.507.308 páginas vistas durante el último mes de medición del año en curso. Tal y como se puede observar en la Tabla 6 , el tercer puesto es ocupado por la versión digital del diario deportivo Marca, que cuenta con 14.867.214 usuarios y 355.972.404 páginas vistas. Algo más abajo en el ranking se sitúan el periódico gratuito 20 minutos, con aproximadamente la mitad de usuarios, y el diario nacional $A B C$, con en torno a los 4 millones.

Tabla 6- Audiencia de medios en Internet. Abril 2009

\begin{tabular}{|c|c|c|c|c|}
\hline Título & URL & Clasificación & $\begin{array}{c}\text { Usuarios } \\
\text { únicos }\end{array}$ & $\begin{array}{c}\text { Páginas } \\
\text { vistas }\end{array}$ \\
\hline SOFTONIC & http://www.softonic.com/ & Informática & 36.967 .521 & 258.933 .710 \\
\hline EL MUNDO & http://www.elmundo.es/ & Noticias e inform & 20.086 .109 & 340.507 .308 \\
\hline MARCA.COM & http://www.marca.com/ & Entretenimiento & 14.867 .214 & 355.972 .404 \\
\hline EMAGISTER & http://www.emagister.com/ & Educ. y Empleo & 12.579 .229 & 50.949 .965 \\
\hline WEBLOGS, S. L. & http://www.weblogssl.com/ & Informática & 9.966 .821 & 43.706 .855 \\
\hline UPTODOWN.COM & http://www.uptodown.com/ & Informática & 9.048 .885 & 49.799 .056 \\
\hline 20MINUTOS.ES & http://www.20minutos.es/ & Noticias e Inform & 7.806 .131 & 101.516 .628 \\
\hline PAGINAS AMARILLAS & http://www.paginasamarillas.es/ & Noticias e Inform & 6.311 .652 & 70.631 .383 \\
\hline SEGUNDA MANO & http://www.segundamano.es/ & Entretenimiento & 5.186 .201 & 294.873 .220 \\
\hline TELECINCO & http://www.telecinco.es/ & Educ. y Empleo & 4.683 .206 & 76.293 .224 \\
\hline
\end{tabular}


Retomando el informe de la Asociación para la Investigación de Medios de Comunicación (2009, p. 86), conviene también hacer referencia a la otra herramienta que más utilizan los internautas en su navegación: los directorios y buscadores. Los cinco más utilizados en 2008 siguen siendo los mismos que en 2007. Google, con un 49,9\% de las menciones, es indudablemente el preferido, y muy por debajo se sitúa Yahoo (16,3\%). A una distancia muy superior están MSN, Terra 0 Altavista. En la Tabla 7 se recogen con detalle estos datos.

Google es también, según el informe Brandz: las 100 marcas más poderosas del mundo de la multinacional Millward Brown, la marca global más valorada económicamente, cifrándose en 100.039 millones de dólares frente a los 86.057 del año pasado. El segundo puesto lo ocupa Microsoft, otra de las grandes marcas del entorno digital, y el tercero la legendaria Coca-Cola.

Otras destacadas marcas tecnológicas como IBM y Apple ostentan la cuarta y sexta posición, respectivamente.

\subsection{El internauta ante la publicidad}

Respecto a la relación del internauta con la publicidad interactiva, hay que subrayar que el uso de estrategias publicitarias intrusivas se encuentra en la base de la actitud reticente que se da en ocasiones y que influye de manera decisiva en la eficacia de las campañas. De acuerdo con el ya referenciado estudio Navegantes en la Red (AIMC, 2009, p. 57), el 57,7\% de los internautas considera que la excesiva presencia de publicidad es el principal problema de la Red, una cifra, en todo caso, ligeramente inferior a la del año anterior, cuando se situaba en el 58\%. Ante esto, el número de navegantes que optan por eludir los mensajes

Tabla 7- Directorio y Buscadores más usados 2008

\begin{tabular}{l|l|c|c|}
\hline & Site (URL) & \% Menciones & \% Menciones acumuladas \\
\hline 1 & www.google.es & 52,1 & 52,1 \\
\hline 2 & www.yahoo.es & 16,5 & 68,6 \\
\hline 3 & www.msn.es & 4,0 & 72,5 \\
\hline 4 & www.terra.es & 2,9 & 75,4 \\
\hline 5 & www.live.com (Incluye www.hotmail.com) & 2,0 & 77,4 \\
\hline 6 & www.altavista.com & 1,8 & 79,3 \\
\hline 7 & www.wikipedia.org & 1,7 & 81,0 \\
\hline 8 & Cuadro de búsqueda de Mozilla & 1,2 & 82,1 \\
\hline 9 & www.youtube.com & 1,0 & 83,1 \\
\hline 10 & www.lycos.es & 0,9 & 84,0 \\
\hline 11 & www.ask.com & 0,7 & 84,6 \\
\hline 12 & Cuadro de búsqueda Internet Explorer & 0,5 & 85,1 \\
\hline 13 & Otros & 14,95 & 100,0 \\
\hline
\end{tabular}


publicitarios es muy destacado; en el Gráfico 4 se puede apreciar cómo el 81\% utiliza sistemas para eliminar el correo no deseado o spam y el 76\% hace lo mismo para no ser molestado por las pop-up.

Por otra parte, en ese mismo estudio (AIMC, 2009, p. 94) se concluye que el $75,5 \%$ de los internautas han adquirido un bien 0 contratado un servicio orientados, motivados o informados por contenidos de la web, mientras que sólo el $24,4 \%$ afirma que son otras fuentes las que más han influido. Se pone en evidencia, por tanto, la actitud receptiva de los consumidores hacia los contenidos que consultan en Internet, a pesar de los molestias que suponen algunas prácticas publicitarias.

Otros datos de interés para conocer la percepción de la publicidad on-line durante 2008 son los de la $17^{\mathrm{a}}$ oleada del NetObserver Europa ${ }^{5}$, que

ha precisado más el contenido de las encuestas respecto a estudios anteriores; ese análisis de la percepción se ha centrado en la segunda oleada en el comportamiento de los usuarios de guías de compra o comparativas de precios y los de sites o blogs de marcas. Las principales conclusiones de esta investigación (www.harrisinteractive. com) dejan patente que los usuarios de sites o blogs de marcas tienen una percepción más positiva sobre la publicidad on-line que los usuarios de regulares de las guías de compra y comparativas de precios (véase Gráficos 5 y 6). Así, entre el $60 \%$ y el $94 \%$ de los primeros considera que para la imagen de marca de una empresa es positivo que se anuncie en Internet. Los usuarios de sites y blogs de marcas también encuentran más creativa e innovadora la publicidad interactiva; los porcentajes se sitúan

Gráfico 4- Programas/ sistemas para evitar la publicidad año 2008 en \%

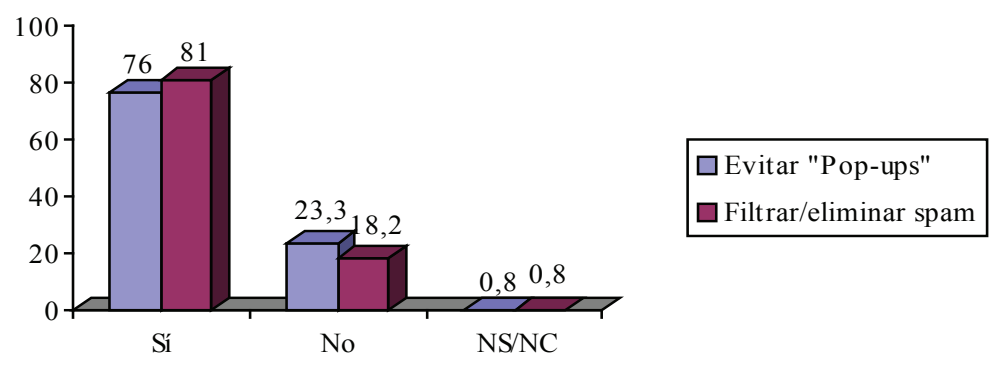

Fuente: AIMC 
en la horquilla del $50 \%$ al $74 \%$. De igual modo, se dan más cuenta de su presencia (entre el 57\% y el 67\%). Otra conclusión relevante es que, salvo el caso de los italianos, entre el 37\% y el 48\% afirma no sentirse molestado por la publicidad on-line. Por el contrario, los usuarios de guías de compra y de comparativas de precios dicen fiarse más de los usuarios antes de comprar un producto o servicio (entre el 58\% y el 74\%). Finalmente, cabe destacar la percepción positiva que, a grandes rasgos, tienen los españoles de la publicidad en Internet, seguidos de italianos y franceses, mientras que en términos generales los alemanes son los más reacios a considerar positivo para la imagen de una marca el hecho de que se anuncie en Internet. Además, a la hora de juzgar la publicidad interactiva por intromisión en la privacidad, los españoles son en general menos críticos que el conjunto de los europeos, a excepción de los italianos y en ocasiones de los franceses.

\section{Conclusiones}

Tras el recorrido que hemos hecho a través de los últimos datos por los diferentes agentes implicados en la industria publicitaria en

Gráfico 5- Percepción y opinión de la publicidad por los usuarios regulares de las guías de compras y de comparativas de precios en Europa en \%, 2008

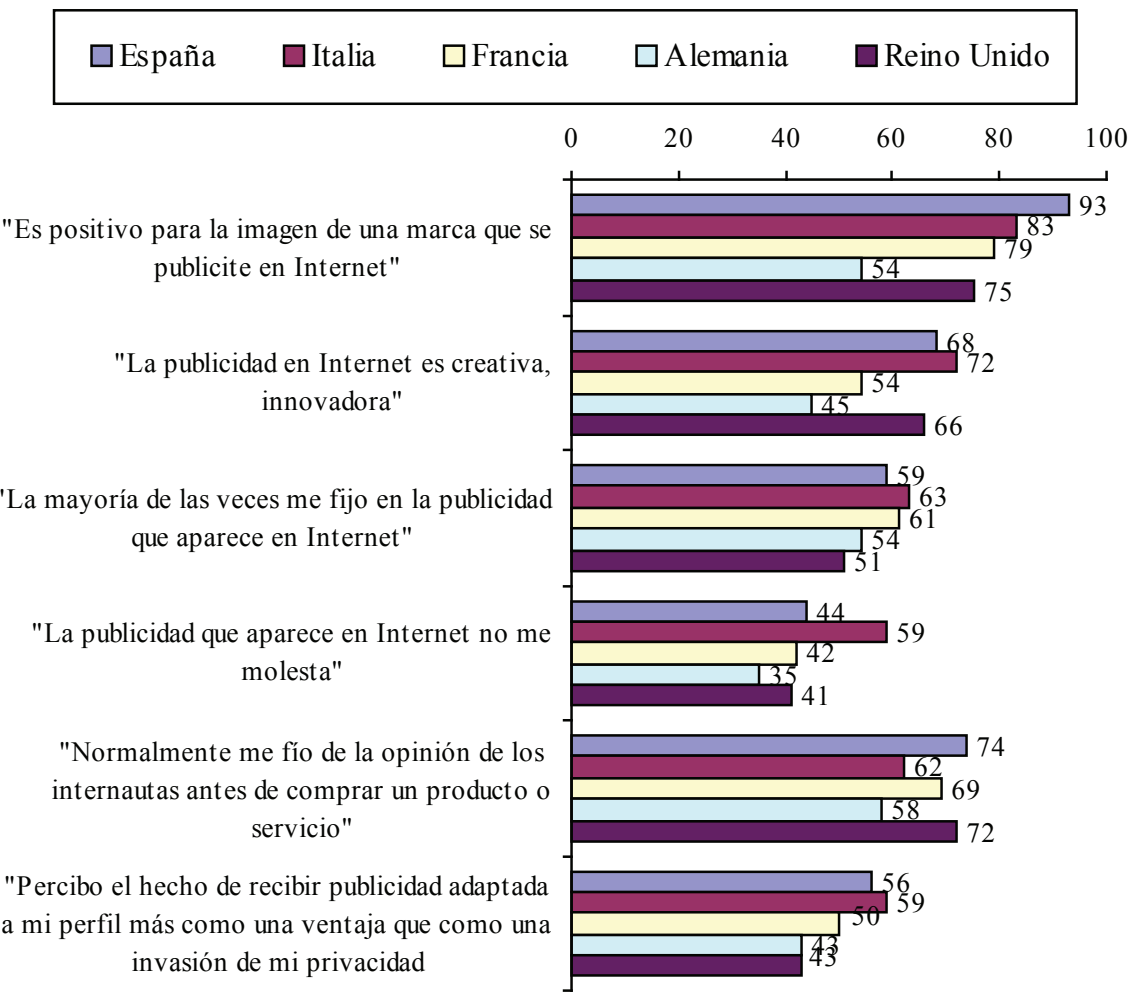


Internet, cabe concluir, una vez más, sobre el incuestionable atractivo que este medio tiene para pequeños y grandes anunciantes; los índices de crecimiento de la inversión en los últimos años son la prueba más palpable de ello. Gracias al potencial interactivo de la Red, los anunciantes son ya conscientes de la importancia de las campañas on-line y, lo que es más importante, de la necesidad de fundamentarlas sobre el respeto a un consumidor cada vez más crítico y con un mayor control sobre los mensajes comerciales que recibe.

Además, la Red ha traído consigo una clara democratización de la publicidad por ser accesible a todo tipo de empresas, desde las pymes hasta las grandes multinacionales; formatos con gran aceptación como los enlaces patrocinados permiten altos índices de eficacia y rentabilidad a anunciantes de toda índole.

Sin duda, a ese éxito también contribuyen los excelentes profesionales de la publicidad interactiva que hay en España y que han llevado a algunas agencias con sede en nuestro país a liderar la creatividad internacional de la publicidad interactiva.

Como contrapartida, y pese al enorme avance experimentado en los últimos años, la publicidad en Internet debe explotar aún más el potencial comunicativo del medio, la gran joya de la interactividad. Diferentes indicadores apuntan, precisamente, a las enormes posibilidades de futuro de una publicidad interactiva cada vez menos intrusiva y más útil para el internauta.

Por último, no podemos olvidar que la integración de los diferentes medios es inevitable y que el concepto de red se amplía y se materializa cada vez más en una creciente diversidad de terminales de recepción; por supuesto los ordenadores, pero también con gran fuerza otros muchos como teléfonos móviles, videoconsolas portátiles o dispositivos de reproducción audiovisual. Por eso, la industria de la publicidad interactiva está asistiendo a la expansión de su campo de actuación para intentar llegar al consumidor allí donde pueda hacerlo de modo más eficaz. Esa eficacia no será posible si no es a través de estrategias respetuosas con el internauta, formas innovadoras y demandadas por los propios consumidores, por lo que esperamos que no se vuelva a caer en la tradicional y molesta insistencia de la publicidad cuyo fin es vencer por redundancia y no por convencimiento.

\section{Referencias}

ASOCIACIÓN PARA LA INVESTIGACIÓN DE MEDIOS DE COMUNICACIÓN. Navegantes en la red: $11^{\mathrm{a}}$ encuesta AIMC a usuarios de Internet. Madrid: AIMC, 2009.

Estudio general de medios: audiencia de internet febrero/marzo de 2009. 2009. Disponible en: $<$ http://www.aimc.es/aimc.php>. Acceso en: 10 ene 2010.

COMSCORE. Russia has fastest growing internet population in Europe. 2008. Disponible en: $<$ http://www.comscore.com/Press_Events/Press_ 
Releases/2008/08/Russia_Internet_Growth $>$. Acceso en: 15 dic 2009.

DOUBLE YOU. El primer concurso de televisión que no sucede en televisión. 2008. Disponible en: $<$ http://festivals.doubleyou.com/2008/atrapalo/ onlinecampaign/elsol.html>. Acceso en: 10 oct 2009.

HARRIS INTERACTIVE. Resultados de la decimoséptima oleada del estudio Net0bserver® Europa. 2008. Disponible en: <http://www. harrisinteractive.fr/services/pubs/HI_FR_ NetObserverEurope_V17_ES_sept08.pdf $>$. Acceso en: 13 ene 2010.

IAB SPAIN. Estudio sobre inversión publicitaria en medios interactivos. 2009. Disponible en: <www. iabspain.net/descargas/descarga.php?id=109>. Acceso en: 15 dic 2009.

IAB UK. 2008: Internet advertising worth over £3.3 billion. 2009. Disponible en: <http://www.iabuk.net/ media/images/Onlineadspendfactsheet-H22008_4293. pdf $>$. Acceso en: 1 ene 2010.

IAB USA. IAB Internet advertising revenue report: 2008 full-year results. 2009. Disponible en: <http:// www.iab.net/media/file/IAB_PwC_2008_full_year. pdf>. Acceso en: 15 feb 2010.

INFOADEX. Estudio Infoadex de la inversión publicitaria en España 2009. Madrid: Infoadex, 2009. MILLWARD BROWN. Branz top: 100 most valuable global brands, 2009. Disponible en: <http://www. brandz.com/upload/brandz-report-2009-completereport(1).pdf>. Acceso en: 20 ene 2010.

MUELA MOLINA, Clara. Estrategias de publicidad interactiva a través de buscadores: los enlaces patrocinados. Telos. Cuadernos de Comunicación e Innovación, Madrid, v. 75, n. 75, p. 59-65, 2008a. La publicidad en Internet: situación actual y tendencias en la comunicación con el consumidor. Zer.
Revista de Estudios de Comunicación, Bilbao, v. 24, n. 24, p. 183-201, 2008b.

\section{OFICINA DE JUSTIFICACIÓN DE LA DIFUSIÓN}

(OJD). 2009. Disponible en: <http://www.

ojdinteractiva.es/alfabetico.php $>$. Acceso en: 20 feb 2010.

THE WON REPORT. 2009. Disponible en: $<$ http:// www.thebigwon.com/wp-content/uploads/2008/09/ wonreport2008presentation.pdf>. Acceso en: 15 feb 2010.

TRAFFICKING SOLUTIONS. Análisis de la publicidad on-line en España: Tendencias y usos. 2009. Disponible en: <http://online-marketing.es/2009/vis/ slides/traffickingsolutions.pps $>$. Acceso en: 1 mar 2010. 


\begin{tabular}{|c|c|}
\hline $\begin{array}{l}\text { A publicidade interativa } \\
\text { na Espanha: situação atual } \\
\text { e tendências }\end{array}$ & $\begin{array}{l}\text { Interactive advertising } \\
\text { in Spain: current situation } \\
\text { and trends }\end{array}$ \\
\hline $\begin{array}{l}\text { Resumo } \\
\text { Este artigo explora a situação atual e tendências da } \\
\text { indústria da publicidade na Internet na Espanha. } \\
\text { Apresenta os dados mais atuais sobre os diversos } \\
\text { agentes envolvidos: o próprio meio, os anunciantes, } \\
\text { as agências, as audiências e os produtos criativos. } \\
\text { Palavras-chave } \\
\text { Anunciantes. Agências interativas. } \\
\text { Publicidade interativa. Internet. Espanha. }\end{array}$ & $\begin{array}{l}\text { Abstract } \\
\text { This article further develops the current situation } \\
\text { and trends of the advertisement industry in the } \\
\text { Internet in Spain. It presents the most updated } \\
\text { information regarding the different agents involved: } \\
\text { the media, advertisers, agencies, audiences and } \\
\text { creative products. } \\
\text { Keywords } \\
\text { Advertisers. Digital agencies. } \\
\text { Interactive advertising. Internet. Spain. }\end{array}$ \\
\hline
\end{tabular}




\section{Expediente}

A revista E-Compós é a publicação científica em formato eletrônico da Associação Nacional dos Programas de Pós-Graduação em Comunicação (Compós). Lançada em 2004, tem como principal finalidade difundir a produção acadêmica de pesquisadores da área de Comunicação, inseridos em instituições do Brasil e do exterior.
E-COMPÓS I www.e-compos.org.br I E-ISSN 1808-2599

Revista da Associação Nacional dos Programas de Pós-Graduação em Comunicação. Brasília, v.13, n.1, jan./abr. 2010.

A identificação das edições, a partir de 2008 passa a ser volume anual com três números.
Afonso Albuquerque

Universidade Federal Fluminense, Brasil

Alberto Carlos Augusto Klein

Universidade Estadual de Londrina, Brasi

Alex Fernando Teixeira Primo

Universidade Federal do Rio Grande do Sul, Brasil

Alfredo Vizeu

Universidade Federal de Pernambuco, Brasil

Ana Carolina Damboriarena Escosteguy

Pontifícia Universidade Católica do Rio Grande do Sul, Brasil

Ana Silvia Lopes Davi Médola

Universidade Estadual Paulista, Brasil

André Luiz Martins Lemos

Universidade Federal da Bahia, Brasil

Ângela Freire Prysthon

Universidade Federal de Pernambuco, Brasil

Antônio Fausto Neto

Universidade do Vale do Rio dos Sinos, Brasil

Antonio Carlos Hohlfeldt

Pontifícia Universidade Católica do Rio Grande do Sul, Brasil

Arlindo Ribeiro Machado

Universidade de São Paulo, Brasil

César Geraldo Guimarães

Universidade Federal de Minas Gerais, Brasil

Cristiane Freitas Gutfreind

Pontifícia Universidade Católica do Rio Grande do Sul, Brasil

Denilson Lopes

Universidade Federal do Rio de Janeiro, Brasil

Eduardo Peñuela Cañizal

Universidade Paulista, Brasil

Erick Felinto de Oliveira

Universidade do Estado do Rio de Janeiro, Brasil

Francisco Menezes Martins

Universidade Tuiuti do Paraná, Brasil

Gelson Santana

Universidade Anhembi/Morumbi, Brasil

Goiamérico Felício

Universidade Federal de Goiás, Brasil

Hector Ospina

Universidad de Manizales, Colômbia

Herom Vargas

Universidade Municipal de São Caetano do Sul, Brasil

leda Tucherman

Universidade Federal do Rio de Janeiro, Brasil

Itania Maria Mota Gomes

Universidade Federal da Bahia, Brasil

Janice Caiafa

Universidade Federal do Rio de Janeiro, Brasil

Jeder Silveira Janotti Junior

Universidade Federal da Bahia, Brasil

\section{CONSELHO EDITORIAL}

João Freire Filho

Universidade Federal do Rio de Janeiro, Brasil

John DH Downing

University of Texas at Austin, Estados Unidos

José Luiz Aidar Prado

Pontifícia Universidade Católica de São Paulo, Brasil

José Luiz Warren Jardim Gomes Braga

Universidade do Vale do Rio dos Sinos, Brasi

Juremir Machado da Silva

Pontifícia Universidade Católica do Rio Grande do Sul, Brasil

Lorraine Leu

University of Bristol, Grã-Bretanha

Luiz Claudio Martino

Universidade de Brasília, Brasil

Maria Immacolata Vassallo de Lopes

Universidade de São Paulo, Brasil

Maria Lucia Santaella

Pontifícia Universidade Católica de São Paulo, Brasil

Mauro Pereira Porto

Tulane University, Estados Unidos

Muniz Sodre de Araujo Cabral

Universidade Federal do Rio de Janeiro, Brasil

Nilda Aparecida Jacks

Universidade Federal do Rio Grande do Sul, Brasil

Paulo Roberto Gibaldi Vaz

Universidade Federal do Rio de Janeiro, Brasil

Renato Cordeiro Gomes

Pontifícia Universidade Católica do Rio de Janeiro, Brasil

Ronaldo George Helal

Universidade do Estado do Rio de Janeiro, Brasil

Rosana de Lima Soares

Universidade de São Paulo, Brasil

Rossana Reguillo

Instituto Tecnológico y de Estudios Superiores do Occidente, México

Rousiley Celi Moreira Maia

Universidade Federal de Minas Gerais, Brasil

Samuel Paiva

Universidade Federal de São Carlos, Brasil

Sebastião Albano

Universidade Federal do Rio Grande do Norte, Brasil

Sebastião Carlos de Morais Squirra

Universidade Metodista de São Paulo, Brasil

Simone Maria Andrade Pereira de Sá

Universidade Federal Fluminense, Brasi

Suzete Venturelli

Universidade de Brasília, Brasil

Valério Cruz Brittos

Universidade do Vale do Rio dos Sinos, Brasil

Veneza Mayora Ronsini

Universidade Federal de Santa Maria, Brasil

Vera Regina Veiga França

Universidade Federal de Minas Gerais, Brasil

\section{COMISSÃO EDITORIAL}

Felipe da Costa Trotta I Universidade Federal de Pernambuco, Brasil Rose Melo Rocha I Escola Superior de Propaganda e Marketing, Brasil CONSULTORES AD HOC

João Maia I Universidade do Estado do Rio de Janeiro, Brasil Sandra Gonçalves I Universidade Federal do Rio Grande do Sul, Brasil Mayra Rodrigues Gomes I Universidade de São Paulo, Brasil Gisela Castro I Escola Superior de Propaganda e Marketing, Brasil João Carrascoza I Escola Superior de Propaganda e Marketing, Brasil Luciana Pellin Mielniczuk I Universidade Federal de Santa Maria, Brasil Irene de Araújo Machado I Universidade de São Paulo, Brasil Hermilio Pereira dos Santos Filho I Pontifícia Universidade Católica, Brasil Benjamim Picado I Universidade Federal Fluminense, Brasil Maria Apaecida Baccega I Escola Superior de Propaganda e Marketing, Brasil Rogério Ferraraz I Universidade Anhembi Morumbi, Brasil

Bruno Souza Leal I Universidade Federal de Minas Gerais, Brasi REVISÃO DE TEXTO E TRADUÇÃo I Everton Cardoso EDITORAÇ̃̃ ELETRÔNICA I Roka Estúdio
COMPóS I www.compos.org.br

Associação Nacional dos Programas de Pós-Graduação em Comunicação

Presidente

Universidade Federal da Bahia, Brasil

itania@ufba.br

Vice-presidente

Julio Pinto

Pontifícia Universidade Católica de Minas Gerais, Brasil juliopinto@pucminas.br

Secretária-Geral

Ana Carolina Escosteguy

Pontifícia Universidade Católica do Rio Grande do Sul, Brasil carolad@pucrs.br
Itania Maria Mota Gomes 